\title{
A Brief Study on Recent Epidemiological Trend of Dengue in ATeriary Care Hospital In Kolkata
}

\author{
Ranadeep Ghosh ${ }^{1}$, JayashreeKonar ${ }^{2}$, KheyaMukherjee ${ }^{1}$, ShivSekharChatterjee ${ }^{1}$, \\ Sipra Saha ${ }^{2}$, Amit Kumar Majumdar ${ }^{1}$, Susmita Bhattacharya ${ }^{3}$ \\ ${ }^{1}$ Assistant Professor (Department of Microbiology, Nil Ratan Sircar Medical College \&Hospital,Kolkata) \\ ${ }^{2}$ Demonstrator(DepartmentofMicrobiology,NilRatanSircarMedicalCollege\&Hospital,Kolkata) \\ ${ }^{3}$ Professor(DepartmentofMicrobiology,NilRatanSircarMedicalCollege \&Hospital,Kolkata)
}

\begin{abstract}
:
Introduction: Dengue fever is a recurrent problem in West Bengal. Dengue Haemorrhagic Fever (DHF) and Dengue Shock Syndrome(DSS) are two potentially fatal complications of Dengue fever.

Objectives:The study was performed to find out the seroprevalence and recent trend of Dengue among the symptomatic patients attending a tertiary care hospital in Kolkata.

Materials \& Methods:A total of 673 suspected cases fulfilling the inclusion criteria were tested by Dengue IgM Capture ELISA over a period of four years (2012-2015) in the department of Microbiology, NilRatanSircar Medical College \& Hospital, Kolkata.

Results:Total 150 samples were found to be Dengue IgM reactive (22.28\%).Amongst the Dengue IgMseropositive cases, 92 cases were male (61.33\%) whereas 58 cases were female (38.67\%). Male predominance was thus observed over the females throughout the study period.Majority of the reactive cases (46\%) were in the age group (10 to 29 years) in all four years of the study period. A clear post rainy season peak was noted in each year of the study period. A peak rise was observed in 2012, then a fall during 2013 and 2014 followed by a sharp rise in 2015.Dengue IgMseropositive cases during the year 2015 on follow uprevealedno report of any death.
\end{abstract}

Conclusion: Strict surveillance and compliance with the vector control programme are required to prevent Dengue outbreak

Key words:Dengue, IgM Capture ELISA, trend

\section{Introduction}

Dengue virus is a mosquito-borne flavivirus and one of the most prevalent arboviruses in tropical and subtropical regions of the world. Dengue Haemorrhagic Fever (DHF) and Dengue Shock Syndrome(DSS) are two potentially fatal complications of Dengue fever.Dengue fever is a recurrent problem in West Bengal. In Kolkata (Calcutta) Dengue was first documented in 1824. Thereafter several epidemics of Dengue were observed in Kolkata in 1836, 1906, 1911and 1923.This epidemic in 1923 wasofsevere degree affecting about $40 \%$ of people. In India, DHF was first reported in Kolkata in 1963-64 where 200 people died. Another small outbreak of DHF occurred in 1990 in Kolkata where 12 children died. In the recent past, there has been a large outbreak involving almost all districts of West Bengal with 6293 cases and 27 deathsin 2005. In Kolkata alone 3967 persons were affected with 14 deaths.This has been followed by smaller number of cases occurring in subsequent years. ${ }^{1}$ With increasing global temperature and associated climatic changes there are apprehensions about occurrence of an outbreak in coming years.

Dengue virus is a positive-stranded encapsulated RNA virus. The genomic RNA is approximately $11 \mathrm{~kb}$ in length and is composed of three structural protein genes that encode the nucleocapsid or core protein $(\mathrm{C})$, a membrane associated protein (M), an envelope protein (E), and seven non-structural (NS) protein genes. The gene order is 5'-CprM (M)-E-NS1-NS2A-NS2B-NS3-NS4A-NS4B-NS5-3', as for other flaviviruses. ${ }^{2}$

Till now, dengue fever was believed to be caused by four different serotypes(DEN-1, DEN-2, DEN-3 and DEN-4). The fifth variant DEN-5 has been isolated in October $2013^{3}$.It has generally been accepted that secondary infection or infection with multiple with dengue virus serotypes is a major risk factor for DHF and DSS due to antibody-dependent enhancement. ${ }^{4}$

Most of the time Dengue is a mild illness but in $<5 \%$ cases, it can be complicated by haemorrhagic complications or shock which can be fatal unless recognized in time and managed efficiently. Complications are more common in children. Sequential infections by different serotypes have been implicated for contributing to complications especially if infection with DEV-1 is followed by DEV-2 or DEV-3 $3^{5}$.

Laboratory diagnosis of dengue virus infection can be made by the detection of specific virus, viral antigen, genomic sequence, and/or antibodies. After the onset of illness, the virus is found in serum or plasma, 
circulating blood cells, and selected tissues, especially those of the immune system, for approximately 2 to 7 days, roughly corresponding to the period of fever.

Traditionally, Dengue is diagnosed on basis of clinical criteria supported by serological report. Dengue specific IgM antibody is classically positive from 5 days of fever onwards in case of primary infection ${ }^{6}$.

The acquired immune response following a Dengue infection consists of the production of IgM and $\mathrm{IgG}$ antibodies primarily directed against the virus envelope proteins. The immune response varies depending on whether the individual has a primary (first dengue or other flavivirus infection) versus a secondary (had Dengue or other flavivirus infection in past) Dengue infection. In general, diagnosis of Dengue is dependent on the phase of the infection. The general timeline of a primaryinfection from virus isolation/identification, to $\operatorname{IgM}$ detection followed by $\mathrm{IgG}$ detection is as follows:

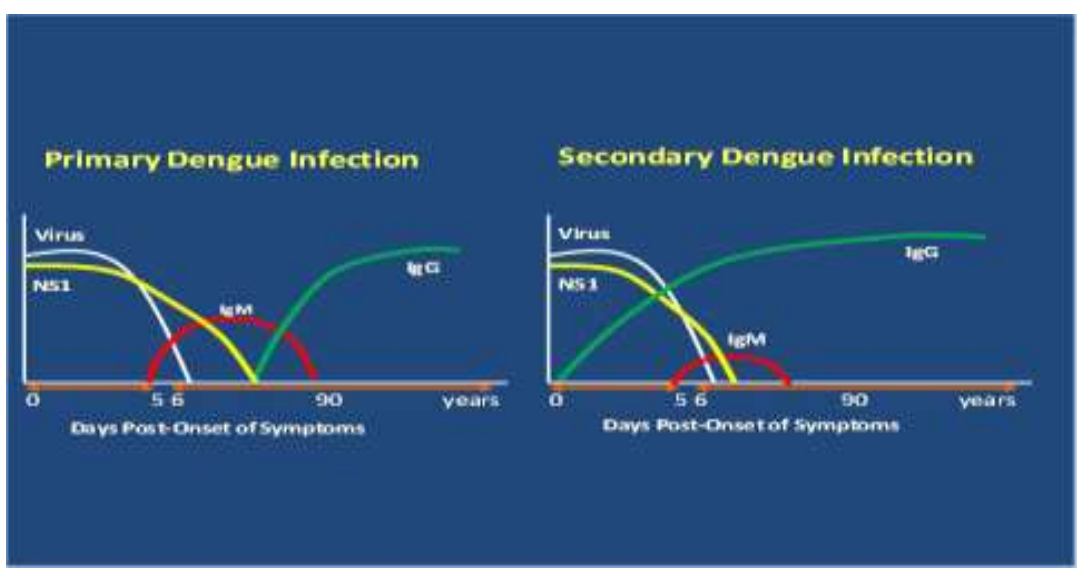

A primary dengue infection is characterized by a slow and low titre antibody response. IgM antibody is the first immunoglobulin isotype to appear. Anti-Dengue IgG is detectable at low titre at the end of the first week of illness, slowly rising thereafter. In contrast, during a secondary infection, antibody titres rise extremely rapidly and antibody reacts broadly with many flaviviruses. High levels of IgG are detectable even in the acute phase and they rise dramatically over the preceding two weeks. The kinetics of the IgM response is more variable. IgM levels are significantly lower in secondary dengue infections and thus some anti-dengue IgM false-negative reactions are observed during secondary infections. According to the Pan American Health Organization (PAHO) guidelines, $80 \%$ of all dengue cases have detectable IgM antibody by day five of illness, and $93-99 \%$ of cases have detectable IgMfor 90 days.

IgM capture ELISA (MAC-ELISA) has become an important tool for routine diagnosis ofdenguevirus infection. Ithasgot a sensitivity and specificity of approximately $90 \%$ and $98 \%$ respectively, but only when used five or more days after onset of fever.

\section{Severe dengue is defined by one or more of the following clinical conditions:}

(i) Plasma leakage that may lead to shock (dengue shock) and/or fluid accumulation, with or without respiratory distress, and/or (ii) Severe bleeding, and/or (iii) Severe organ impairment.

Severe dengue should be considered if the patient is coming from an area considered as dengue risk zone,presenting with fever of 2-7 days plus any of the following features:

\section{- There is evidence of plasma leakage, such as:}

- High or progressively rising haematocrit;

- Pleural effusions or ascites;

- Circulatory compromise or shock (tachycardia, cold and clammy extremities, capillary refill time greater than three seconds, weak or undetectable pulse, narrow pulse pressure or, in late shock andunrecordable blood pressure).

- There is significant bleeding.

- There is an altered level of consciousness (lethargy or restlessness, coma, convulsions)

- There is severe gastrointestinal involvement (persistent vomiting, increasing or intense abdominal pain, jaundice)

- There is severe organ impairment (acute liver failure, acute renal failure, encephalopathy or encephalitis, or other unusual manifestations, cardiomyopathy) or other unusual manifestations. 
Severe manifestations develop in less than 5\% cases. All patients with severe dengue should be admitted to a hospital with access to intensive care facilities and blood transfusion. Judicious intravenous fluid resuscitation is the most essential and usually the onlyintervention required. Dengue Haemorrhagic Fever cases may need platelet, plasma or blood transfusion. Inspite of taking all these measures, still there can be significant mortality and morbidity if Classical dengue fever is associated with complications of DHF or DSS.

Co-infection of Dengue and Chikungunya or Dengue and Zika yields a grave prognosis. ${ }^{7}$

Prediction of impending severity in a dengue patient before they actually develop may help in timely interventions and averting morbidity and mortality.

Laboratory Criteria for Diagnosis of Dengue are as follows ${ }^{8}$ :

\section{- Confirmed:}

- Detection of DENV nucleic acid in serum, plasma, blood, cerebrospinal fluid (CSF), other body fluid or tissue by validated reverse transcriptase-polymerase chain reaction (PCR), or

- Cell culture isolation of DENV from a serum, plasma, or CSF specimen; or

- Detection of IgM anti-DENV by validated immunoassay in a serum specimen or CSF in a person living in a dengue endemic or non-endemic area of the United States without evidence of other flavivirus transmission (e.g., West Nile Virus, Japanese EncephalitisVirus or recent vaccination against a flavivirus (e.g., Yellow Fever Virus, Japanese Encephalitis Virus); or

- Detection of IgM anti-DENV in a serum specimen or CSF by validated immunoassay in a traveller returning from a dengue endemic area without on-going transmission of another flavivirus (e.g., West Nile Virus, JapaneseEncephalitisVirus), clinical evidence of co-infection with one of these flaviviruses, or recent vaccination against a flavivirus (e.g., Yellow Fever Virus, Japanese Encephalitis Virus); or

- IgM anti-DENV seroconversion by validated immunoassay in acute (i.e., collected $<5$ days of illness onset) and convalescent (i.e., collected $>5$ days after illness onset) serum specimens; or

- IgG anti-DENV seroconversion or $\geq 4$-fold rise in titre by a validated immunoassay in serum specimens collected $>2$ weeks apart, and confirmed by a neutralization test (e.g., plaque reduction neutralization test) with a $>4$-fold higher end point titre as compared to other flaviviruses tested.

- Probable:

- Detection of IgM anti-DENV by validated immunoassay in a serum specimen or CSF in a person living in a dengue endemic or non-endemic area of the United States with evidence of other flavivirus transmission (e.g., Yellow Fever Virus, Japanese Encephalitis Virus), or recent vaccination against a flavivirus (e.g., Yellow Fever Virus, Japanese Encephalitis Virus).

- Detection of IgM anti-DENV in a serum specimen or CSF by validated immunoassay in a traveller returning from a dengue endemic area with on-going transmission of another flavivirus (e.g., West Nile Virus, Yellow Fever Virus, Japanese Encephalitis Virus), clinical evidence of co-infection with one of these flaviviruses, or recent vaccination against a flavivirus (e.g., Yellow Fever Virus, Japanese Encephalitis Virus).

- Suspected:

- The absence of IgM anti-DENV by validated immunoassay in a serum or CSF specimen collected $<5$ days after illness onset and in which molecular diagnostic testing was not performed in a patient with an epidemiologic linkage.

D dimer (DD) has been found to be more significantly [p<0.03] present in the DHF group (87\%) than in the DF group (13\%). The sensitivity and specificity of DD in predicting severe dengue infection (DHF) were $90 \%$ and $67 \%$, respectively. Sequential analysis of DD showed higher levels at all stages of dengue infection. It correlated with the disease severity. ${ }^{9}$

IL-1 $\beta$, IFN- $\gamma$, IL-4, IL-6, IL-13, IL-7 and GM-CSF were significantly increased in patients with severe clinical manifestations (severe dengue) when compared to mild disease forms (mild dengue). In contrast, increased MIP-1 $\beta$ levels were observed in patients with mild dengue. MIP-1 $\beta$ was also associated with CD56+NK cell circulating rates. IL-1 $\beta$, IL-8, TNF- $\alpha$ and MCP-1 were associated with marked thrombocytopenia. Increased MCP-1 and GM-CSF levels correlated with hypotension. Moreover, MIP-1 $\beta$ and IFN- $\gamma$ were independently associated with both dengue fever severity and disease outcome. ${ }^{10}$

For prevention of Dengue, vector control and integrated surveillance is suggested in India as till date no effective vaccine is recommended.

Vector control- The National Vector Borne Disease Control Program has been initiated to control the emerging threat by vector-borne diseases in India. A. aegypti is the commonest vector of dengue in India, 
followed by A. albopictus. Vector control methods involve environmental, chemical, and biological management approaches. Utilizing an effective integrated vector control strategy with a combination of approaches, such as social mobilization and integration of chemical and nonchemical vector control methods targeting areas of high human-vector contact, will aid in reducing dengue transmission. The evolution and spread of resistance to insecticides is a growing concern for the control of dengue vectors. Bioassay data demonstrate that resistance to organophosphates and pyrethroids is widespread among A. aegypti, and resistance has also been reported among A. albopictus. Monitoring of resistance development is, therefore, necessary to ensure that effective insecticides are being used.

Biological control is another helpful measure to break the transmission cycle.

Integrated surveillance - Surveillance is an essential component of any dengue prevention and control program, as it provides the necessary information for risk assessment and program guidance. Surveillance includes both passive and active data collection methodologies ${ }^{11}$.

\section{Objectives:}

1. To find out the seroprevalence of Dengue among the symptomatic patients attending a tertiary care hospital in Kolkata.

2. Documentation of the present scenario and recent trend of Dengue among the study population.

\section{Materials \& Methods}

The study was performed in the department of Microbiology, NilRatanSircar Medical College \& Hospital, Kolkata over a period of four years (From January 2012 to December 2015) with six hundred and seventy three clinically suspected patients attending this tertiary care centrehaving fever for at least five days with the clinical features as mentioned under the inclusion criteria.Blood was collected aseptically from suspected cases, serum was separated and analysed by IgMCapture ELISA technique to detect Dengue specific IgM antibodies.

\section{Inclusion criteria were as follows:}

Fever of $\geq 5$ days with two or more of the following symptoms-

Rash, body ache, malaise, myalgia, arthralgia,retro orbital pain and eye congestion.

Known immunocompromised patients were excluded from the study.

Informed consent was taken from the cases before including them in the study.

There was no ethical controversy and conflict of interest.

\section{Results}

A total of 673 suspected cases were tested by Dengue IgM Capture ELISA over last four years (2012$2015)$ and 150 samples were found to beDengueIgM reactive $(22.28 \%)$.

Amongst the total 150 Dengue IgMseropositive cases, 92 cases were male $(61.33 \%)$ whereas 58 cases were female (38.67\%). Male predominance wasthus observed over female throughout the study period (Figure-1).

Majority of the reactive cases $(46 \%)$ were in the age group (10 to 29 years)as found in each of the four years of the study period(Figure-2).

A clear post rainy season peak was noted in each year (Figure-3).

A peak level of prevalence was observed in 2012, then a fall during 2013 and 2014 followed by a sharprise in 2015 was noted (Figure-4).

Dengue IgMseropositive cases of the year 2015 were followed up when no incidence of any death was being reported.

Figure-1: Gender distribution of Dengue IgMseropositive cases 


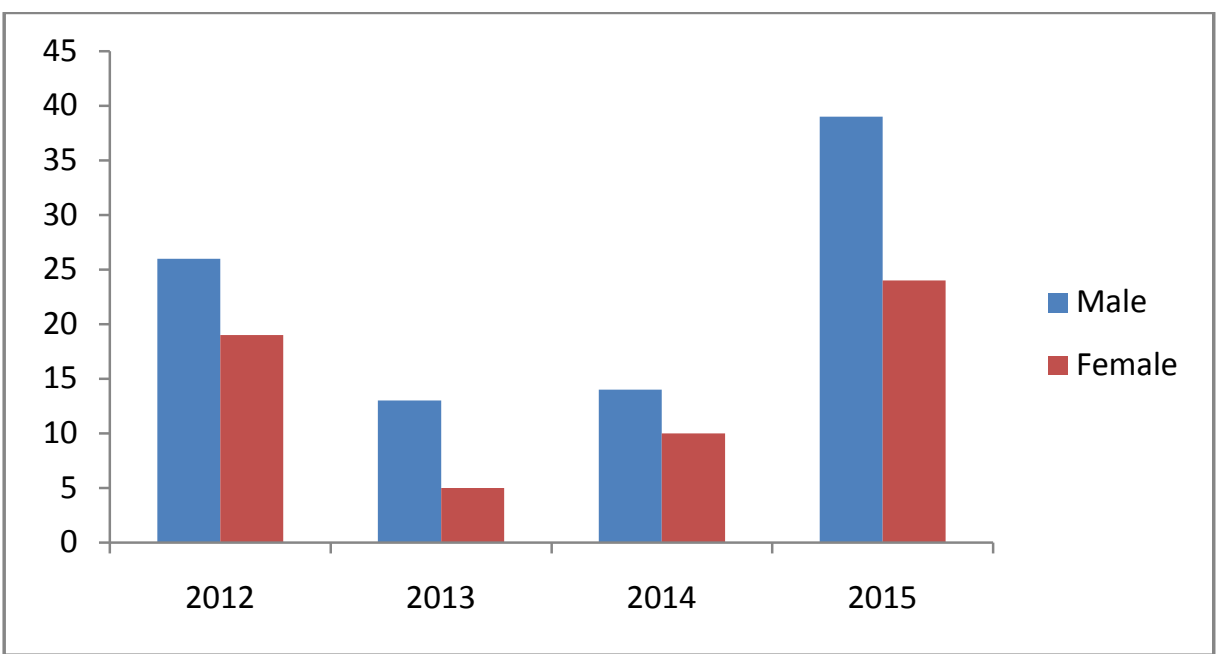

Figure-2: Age wise distribution of Dengue IgMseropositive cases

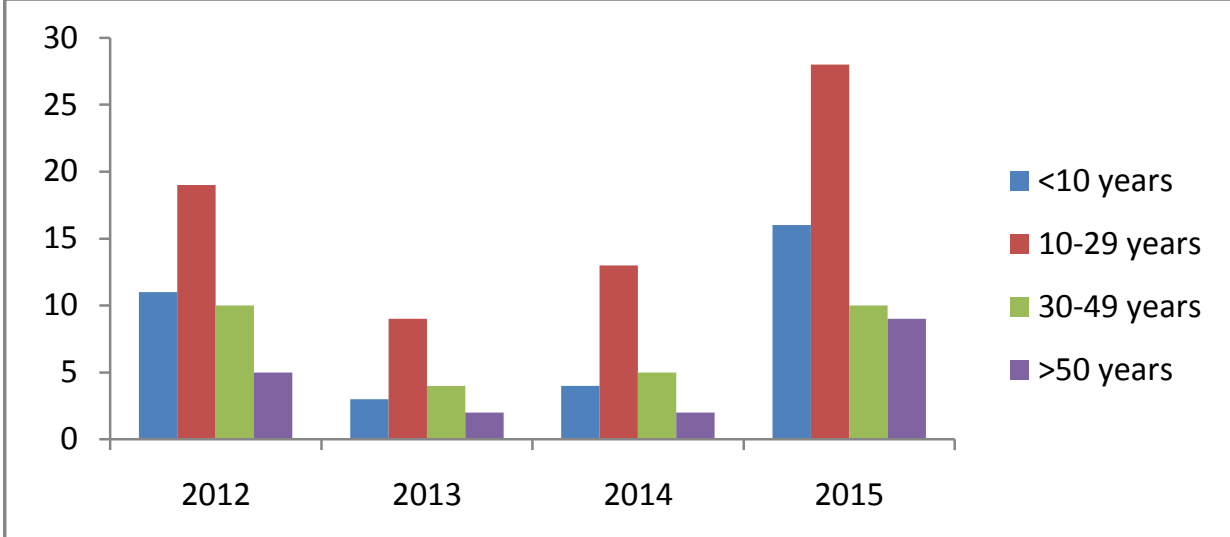

Figure-3: Seasonal distribution of Dengue IgMseropositive cases

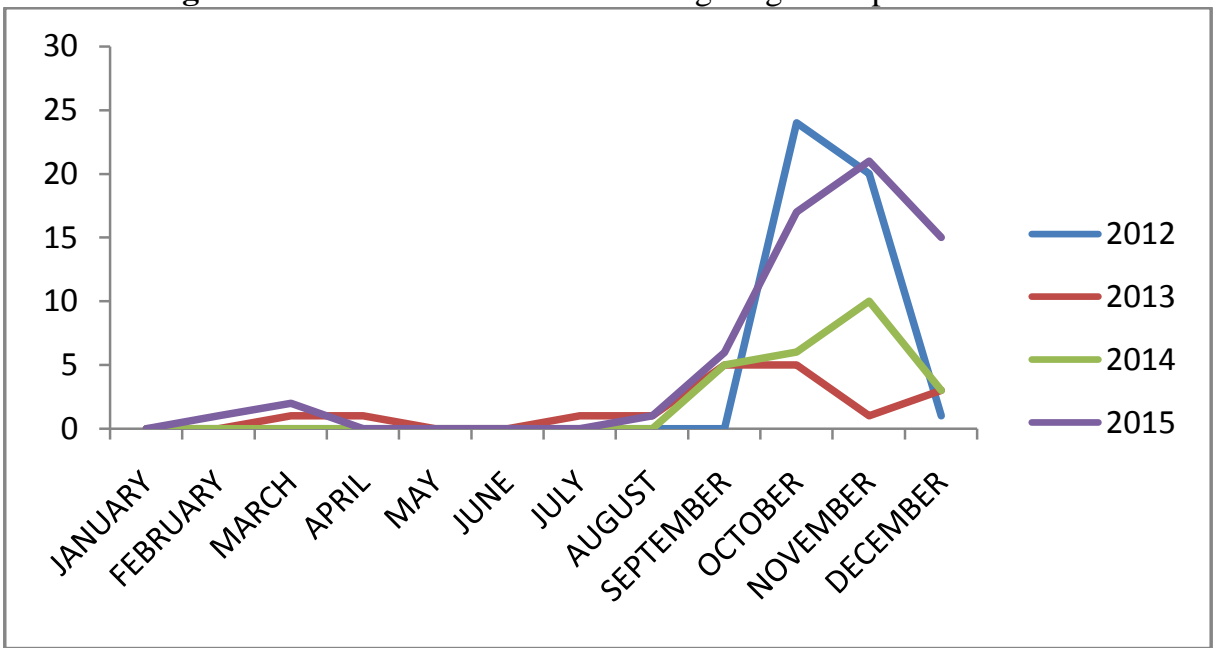

Figure-4: Year wise distribution of Dengue IgMseropositive cases. 




\section{Discussion}

The present study had concentrated on the epidemiology and trend of Dengue fever in a tertiary care hospital of Kolkata, since 2012.

In this study, the highest number of Dengue IgMseropositive cases (46\%) was recorded in the age group (10-29 years) with male preponderance. Findings of Gupta et $a l^{12}$ andChakravarti and Kumaria ${ }^{13}$ were similar to our studyi.e., maximum cases was in the age group (21-30 years) and in the finding that the male patients clearly outnumberingthe female patients. However Sarkaret $a l^{14}$ reported that maximum cases were in the age group $\leq 10$ years and there was female preponderance in their study.

The majority of the cases were reported during the post-monsoon seasons (August to November) with a peak during October and November in all the four years (2012-2015), in accordance with the established findings by P. Reiter. ${ }^{15}$

Our study has revealed the fact that there was a dengue outbreak in West Bengal in 2012 and 2015. All the 63 Dengue IgMseropositive cases of the year 2015 included in our study populationwas followed up and not a single case of death was reported. Our finding in this study corroborates with the findings of Hati ${ }^{16}$, Taraphdaret $a l^{17}$, and Sarkaret al. ${ }^{18}$

\section{Summary \& Conclusion}

The present study was based on the epidemiology and trend of Dengue fever in a tertiary care hospital of Kolkata since 2012.

The study was performed in the department of Microbiology, NRS Medical College \&Hospital,Kolkata over a period of four years from January 2012 to December 2015 with a total of 673 clinically suspected patients attending this institutewho were suffering from fever for at least five days and fulfilled the inclusion criteria.Blood was collected aseptically from the cases, serum was separated and analysed by IgMCapture ELISA technique to detect Dengue specific IgM antibodies. Known immunocompromised cases were excluded from the study.

Out of the 673 suspected cases, 150 samples were found to be Dengue IgM reactive (22.28\%) A total of 92 cases were male $(61.33 \%)$ whereas 58 cases were female $(38.67 \%)$. A male predominance is thus observed over the female cases throughout the study period. Majority of the cases $(46 \%)$ were in the age group of (10 to 29 years). A clear post rainy season peak was noted in each year of the study period (2012-2015). There was a rise in 2012, then a fall in 2013-2014 and again a sharp riseofthe number of Dengue cases was recorded in 2015. These two Dengue outbreaks of 2012 and 2015 reached the peak level in post rainy season months.

Strict surveillance and compliance with the vector control programme are required to prevent Dengue outbreak as no effective vaccine or chemoprophylaxis against Dengueisavailabletill date.

\section{Limitations of the study:}

Haemagglutination inhibition test or Complement Fixation test with the preserved Dengue IgM reactive sera are to be performed for Dengue serotyping. This will help to identify the predominant Dengue serotypes circulating in this region in order to predict the occurrence of complications like Dengue Haemorrhagic Fever (DHF) and Dengue Shock Syndrome (DSS).

\section{Acknowledgements}


The authors express sincere gratitude to the Principal, Dean of students' affairs and Head of the Department of Microbiology of Nil RatanSircar Medical College \& Hospital, Kolkata for their active support and co-operation throughout the study period. The authors specially extend their thanks to the staff of department of Microbiology and all participants included in this study.

\section{References}

[1]. Hati AK. Studies on dengue and dengue haemorrhagic fever (DHF) in West Bengal State, India.JCommun Dis. 2006 Mar;38 (2):124-9.

[2]. Leyssen, P., E. D. Clercq, and J. Neyts. 2000. Perspectives for the treatment of infections with Flaviviridae. Clin. Microbiol. Rev. $13: 67-82$

[3]. Lt Col M.S. Mustafa, Col V. Rasotgi, Col S. Jain Lt Col V. Gupta Discovery of fifth serotype of dengue virus (DENV-5): A new public health dilemma in dengue control. MJAFI 2015 Volume 71, Issue 1, Pages 67-70

[4]. Halstead, S. B. 1988. Pathogenesis of dengue: challenge to molecular biology. Science239:476-481.

[5]. Mourya DT, Yadav P. Vector biology of dengue and chikungunya viruses. Indian J Med Res 2006; 124: 475-80.

[6]. World Health Organization. 1997. Dengue haemorrhagic fever: diagnosis, treatment, prevention and control, 2nd ed. World Health Organization, Geneva, Switzerland

[7]. CaoLormeau VM, Roche C, Musso D, Mallet HP, Dalipanda T, Dofai A, Dengue virus type 3, South Pacific Islands, 2013. Emerg Infect Dis.2014;20:1034-6.

[8]. Www.who.int

[9]. Setrkraising K, Bongsebandhu-phubhakdi C, Voraphani N, Pancharoen C, Thisyakorn U, Thisyakorn C. D-dimer as an indicator of dengue severity. Asian Biomedicine .2007. Vol. 1 No. 1. 54-57.

[10]. Bozza F A, Cruz O G, Zagne S M O, Azeredo E L, Nogueira R M R, Assis E F, Bozza P T, Kubelka C F. Multiplex cytokine profile from dengue patients: MIP-1beta and IFN-gamma as predictive factors for severity. BMC Infectious Diseases. $2008,8: 86$

[11]. Ekta Gupta and NehaBallani.Current perspectives on the spread of dengue in India. Infect Drug Resist. 2014; 7: 337-342

[12]. E. Gupta, L. Dar, G. Kapoor, and S. Broor, "The changing epidemiology of dengue in Delhi, India," Virology Journal, vol. 3, article 92, 2006.

[13]. A. Chakravarti and R. Kumaria, "Eco-epidemiological analysis of dengue infection during an outbreak of dengue fever, India," Virology Journal, vol. 2, article 32, 2005

[14]. A. Sarkar, D. Taraphdar, and S. Chatterjee, "Molecular typing of dengue virus circulating in Kolkata, India in 2010," Journal of Tropical Medicine, vol. 2012, Article ID 960329, 5 pages, 2012.

[15]. P. Reiter, "Climate change and mosquito-borne disease," Environmental Health Perspectives, vol. 109, supplement 1, pp. 141$161,2001$.

[16]. A. K. Hati, "Dengue serosurveillance in Kolkata, facing an epidemic in West Bengal, India," Journal of Vector Borne Diseases, vol. 46, no. 3, pp. 197-204, 2009.

[17]. D. Taraphdar, A. Sarkar, M. K. Bhattacharya, and S. Chatterjee, "Sero diagnosis of dengue activity in an unknown febrile outbreak at the Siliguri Town, District Darjeeling, West Bengal," Asian Pacific Journal of Tropical Medicine, vol. 3, no. 5, pp. 364-366, 2010.

[18]. A. Sarkar, D. Taraphdar, and S. Chatterjee, "Investigations of recurrent outbreaks of unknown fever, establish rural dengue activity in West Midnapore, a costal district in West Bengal, India,” Archives of Clinical Microbiology, vol. 1, no. 4, 2010. 